\title{
Lymph node yield after rectal resection in patients treated with neoadjuvant radiation for rectal cancer: a systematic review and meta-analysis
}

Robert Mechera a,b, Tibor Schuster ${ }^{\mathrm{c}}$, Robert Rosenberg ${ }^{\mathrm{a}}$, Benjamin Speich ${ }^{\mathrm{a},{ }^{*}}$

a Department of Surgery, Cantonal Hospital Baselland, Liestal, Switzerland

${ }^{b}$ Department of Surgery, University Hospital of Basel, Basel, Switzerland

c Department of Family Medicine, McGill University, Montreal, Quebec, Canada

\section{Summary}

Background: The lymph node status $(\mathrm{pN})$ represents a major prognostic factor in colorectal cancer. However, it was demonstrated that neoadjuvant chemoradiotherapy decreases the numbers of lymph nodes in the specimen. The prognostic importance of the number of metastatic lymph nodes in relation to the retrieved remains unclear after radiation. This meta-analysis quantifies the influence of neoadjuvant chemoradiotherapy or radiotherapy on the lymph node yield in rectal cancer patients. Methods: We performed a systematic review and searched PubMed, Embase and the Cochrane Library without any language restriction from first of January 1980 until 31 ${ }^{\text {st }}$ March 2015. Two reviewers examined all publications independently and extracted the relevant data if the study assessed lymph node counts or positive lymph node yields of patient who received neoadjuvant treatment compared to patients who did not receive neoadjuvant treatment. Meta-analyses were conducted to quantify the mean difference in lymph node yield.

Results: A total of 34 articles (including 37 datasets) were included in the metaanalyses. Neoadjuvant chemoradiotherapy resulted in a mean reduction of 3.9 lymph nodes $(95 \% \mathrm{Cl} 3.7$ to 4.1$)$ and an average reduction in harvested positive lymph nodes of 0.7 ( $95 \% \mathrm{Cl} 0.2$ to 1.2 ) compared to patients who received no neoadjuvant therapy. 
Individuals who received neoadjuvant radiotherapy had, in average, 2.1 lymph node less (95\% $\mathrm{Cl} 1.7$ to 2.5$)$ resected compared to their counterparts who received no neoadjuvant treatment.

Conclusions: Neoadjuvant chemoradiotherapy or radiotherapy only in rectal cancer patients leads to a decrease in lymph node harvest of approximately four and two lymph nodes, respectively. Therefore, it is challenging to reach the requirement of the current guideline of twelve resected lymph nodes. We therefore stress the importance of intensifying all efforts from involved subspecialities (i.e. surgeons and pathologists) to reach the benchmark harvest 12 resected lymph nodes according to current guidelines.

Funding: None 
